\title{
Heisenberg's original derivation of the uncertainty principle and its universally valid reformulations
}

\author{
Masanao Ozawa \\ Graduate School of Information Science, Nagoya University, Chikusa-ku, Nagoya, 464-8601, Japan*
}

\begin{abstract}
Heisenberg's uncertainty principle was originally posed for the limit of the accuracy of simultaneous measurement of non-commuting observables as stating that canonically conjugate observables can be measured simultaneously only with the constraint that the product of their mean errors should be no less than a limit set by Planck's constant. However, Heisenberg with the subsequent completion by Kennard has long been credited only with a constraint for state preparation represented by the product of the standard deviations. Here, we show that Heisenberg actually proved the constraint for the accuracy of simultaneous measurement but assuming an obsolete postulate for quantum mechanics. This assumption, known as the repeatability hypothesis, formulated explicitly by von Neumann and Schrödinger, was broadly accepted until the 1970s, but abandoned in the 1980 s, when completely general quantum measurement theory was established. We also survey the author's recent proposal for a universally valid reformulation of Heisenberg's uncertainty principle under the most general assumption on quantum measurement.
\end{abstract}

Keywords: quantum measurement, uncertainty principle, simultaneous measurement, repeatability hypothesis, instruments, root mean square error

\section{INTRODUCTION}

The uncertainty principle proposed by Heisenberg 1 in 1927 revealed that we cannot determine both position and momentum of a particle simultaneously in microscopic scale as stating "the more precisely the position is determined, the less precisely the momentum is known, and conversely"1 and had overturned the deterministic world view based on the Newtonian mechanics. By the famous $\gamma$ ray microscope thought experiment Heisenberg $\frac{1}{2}$ derived the relation

$$
\varepsilon(\hat{q}) \varepsilon(\hat{p}) \sim h
$$

for $\varepsilon(\hat{q})$, the "mean error" of the position measurement, and $\varepsilon(\hat{p})$, thereby caused "discontinuous change" of the momentum, or more generally the mean error of the simultaneous momentum measurement, where $h$ is Planck's constant:

Let $\varepsilon(\hat{q})$ [originally, $q_{1}$ ] be the precision with which the value $q$ is known $(\varepsilon(\hat{q})$ is, say, the mean error of $q$ ), therefore here the wavelength of the light. Let $\varepsilon(\hat{p})$ [originally, $p_{1}$ ] be the precision with which the value $p$ is determinable; that is, here, the discontinuous change of $p$ in the Compton effect $\frac{1}{1}$ (p. 64).

Heisenberg claimed that this relation is a "straightforward mathematical consequence" -1 (p. 65) of fundamental postulates for quantum mechanics. In his mathematical derivation of relation (1), he derived

$$
\sigma(\hat{q}) \sigma(\hat{p})=\frac{\hbar}{2}
$$

for standard deviations $\sigma(\hat{q})$ and $\sigma(\hat{p})$ of position $\hat{q}$ and momentum $\hat{p}$ for a class of Gaussian wave functions, later known as minimum uncertainty wave packets. Subsequently, Kennard 2 proved the inequality

$$
\sigma(\hat{q}) \sigma(\hat{p}) \geq \frac{\hbar}{2}
$$

for arbitrary wave functions. By this relation, the lower bound of relation (11) was later set as

$$
\varepsilon(\hat{q}) \varepsilon(\hat{p}) \geq \frac{\hbar}{2},
$$

where $\hbar=h /(2 \pi)$.

Text books ${ }^{3-6}$ up to the 1960s often explained that the physical meaning of Heisenberg's uncertainty principle is expressed by Eq. (4), but it is formally expressed by Eq. (3). This explanation is later considered to be confusing. In fact, it was said that Eq. (4) expresses a limitation of measurements, while mathematically derived relation Eq. (3) expresses a statistical property of quantum state, or a limitation of state preparations, so that they have different meanings ${ }^{7}$. Thus, Heisenberg with the subsequent completion by Kennard has long been credited only with a constraint for state preparation represented by Eq. (3).

This paper aims to resolve this long standing confusion. It will be shown that Heisenberg ${ }^{1}$ in 1927 actually "proved" not only Eq. (2) but also Eq. (1) from basic postulates for quantum mechanics. In showing that, it is pointed out that as one of the basic postulates Heisenberg supposed an assumption called the "repeatability hypothesis", which is now considered to be obsolete. In fact, in the 1930's the repeatability hypothesis was explicitly claimed by von Neumann $n^{\frac{3}{2}}$ and Schrödinger ${ }^{8}$, whereas this hypothesis was abandoned in the 1980s, when quantum measurement theory was establish to be general enough to treat all the physically realizable measurements.

Through those examinations it will be concluded that Heisenberg's uncertainty principle expressed by Eq. (4) is logically a straightforward consequence of Eq. (3) under a generalized form of the repeatability hypothesis. In fact, under the repeatability hypothesis a measurement is required to prepare the state with a sharp value of the measured observable, and hence the "measuremental" uncertainty relation (4) is a logical consequence of the "preparational" uncertainty relation 3 .

As stated above, the repeatability hypothesis was abandoned in the 1980s, and nowadays relation (4) is taken to be 
a breakable limit ${ }^{9,10}$. Naturally, the problem remains: what is the unbreakable constraint for simultaneous measurements of non-commuting observables? To answer this question, we will survey the author's recent proposal ${ }^{11-13}$ for a universally valid reformulation of Heisenberg's uncertainty principle under the most general assumption on quantum measurement.

\section{REPEATABILITY HYPOTHESIS}

The uncertainty principle was introduced by Heisenberg in a paper entitled Über den anschaulichen Inhalt der quantentheoretischen Kinematik und Mechanik ${ }^{1}$ published in 1927. In what follows we shall examine Heisenberg's derivation of the uncertainty principle following this paper.

Before examining the detail of Heisenberg's derivation, we shall examine the basic postulates for quantum mechanics in Heisenberg's time, following von Neumann's formulation ${ }^{3}$. In what follows, a positive operator on a Hilbert space with unit trace is called a density operator. We denote by $\mathscr{B}(\mathbb{R})$ the set of Borel subsets of $\mathbb{R}$ and by $E^{A}$ the spectral measure of a self-adjoint operator $A$, i.e., $A$ has the spectral decomposition $A=\int_{\mathbb{R}} \lambda E^{A}(d \lambda)$.

Axiom 1 (States and observables). Every quantum system $\mathbf{S}$ is described by a Hilbert space $\mathscr{H}$ called the state space of $\mathbf{S}$. States of $\mathbf{S}$ are represented by density operators on $\mathscr{H}$ and observables of $\mathbf{S}$ are represented by self-adjoint operators on $\mathscr{H}$.

Axiom 2 (Born statistical formula). If an observable $A$ is measured in a state $\rho$, the outcome obeys the probability distribution of $A$ in $\rho$ defined by

$$
\operatorname{Pr}\{A \in \Delta \| \rho\}=\operatorname{Tr}\left[E^{A}(\Delta) \rho\right],
$$

where $\Delta \in \mathscr{B}(\mathbb{R})$.

Axiom 3 (Time evolution). Suppose that a system $\mathbf{S}$ is an isolated system with the (time-independent) Hamiltonian $H$ from time t to $t+\tau$. The system $\mathbf{S}$ is in a state $\rho(t)$ at time $t$ if and only if $\mathbf{S}$ is in the state $\rho(t+\tau)$ at time $t+\tau$ satisfying

$$
\rho(t+\tau)=e^{-i \tau H / \hbar} \rho(t) e^{i \tau H / \hbar}
$$

Under the above axioms, we can make a probabilistic prediction of the result of a future measurement from the knowledge about the past state. However, such a prediction applies only to a single measurement in the future. If we make many measurements successively, we need another axiom to determine the state after each measurement. For this purpose, the following axiom was broadly accepted in the 1930s.

Axiom 4 (Measurement axiom). If an observable A is measured in a system $\mathbf{S}$ to obtain the outcome a, then the system $\mathbf{S}$ is left in an eigenstate of A belonging to $a$.

Von Neuamann ${ }^{3}$ showed that this assumption is equivalent to the following assumption called the repeatability hypothesi $\mathrm{s}^{3}$ (p. 335), posed with a clear operational condition generalizing a feature of the Compton-Simons experiment ${ }^{3}$ (pp. 212-214).

(R) Repeatability hypothesis. If an observable A is measured twice in succession in a system $\mathbf{S}$, then we get the same value each time.

It can be seen from the following definition of measurement due to Schrödinger given in his famous "cat paradox" paper 8 that von Neumann's repeatability hypothesis was broadly accepted in the 1930s.

The systematically arranged interaction of two systems (measured object and measuring instrument) is called a measurement on the first system, if a directly-sensible variable feature of the second (pointer position) is always reproduced within certain error limits when the process is immediately repeated (on the same object, which in the meantime must not be exposed to any additional influences) $)^{8}$.

Based on the repeatability hypothesis von Neumann ${ }^{3}$ proved the impossibility of simultaneous measurement of two non-commuting observables as follows. Suppose that two observables $A, B$ are simultaneously measurable in every state and suppose that the eigenvalues of $A$ are non-degenerate. Then, the state just after the simultaneous measurement of $A$ and $B$ is a common eigenstate of $A$ and $B$, so that there is an orthonormal basis consisting of common eigenstates of $A$ and $B$, concluding that $A$ and $B$ commute.

Since Heisenberg's uncertainty principle concerns measurements with errors, it is naturally expected that it can be mathematically derived by extending the above argument to approximate measurements.

\section{APPROXIMATE REPEATABILITY HYPOTHESIS}

To extend the repeatability hypothesis to approximate measurements, we generalize the notion of eigenstates as follows. For any real number $\lambda$ and a positive number $\varepsilon$, a (vector) state $\psi$ is called an $\varepsilon$-approximate eigenstate belonging to $\lambda$ iff the relation

$$
\|A \psi-\lambda \psi\| \leq \varepsilon
$$

holds. If $\varepsilon=0$, the notion of $\varepsilon$-approximate eigenstates is reduced to the ordinary notion of eigenstates. A real number $\lambda$ is called an approximate eigenvalue of an observable $A$ iff for every $\varepsilon>0$ there exists an $\varepsilon$-approximate eigenstate of $A$. The set of approximate eigenvalues of an observable $A$ coincides with the spectrum of $A^{14}$ (p. 52).

Now, we formulate the approximate repeatability hypothesis as follows.

(AR) Approximate Repeatability Hypothesis. If an $o b$ servable $A$ is measured in a system $\mathbf{S}$ with mean error $\varepsilon$ 
to obtain the outcome a, then the system $\mathbf{S}$ is left in an $\varepsilon$ approximate eigenstate of $A$ belonging to $a$.

Obviously, (AR) is reduced to (R) for $\varepsilon=0$. Since we have

$$
\|A \psi-\lambda \psi\| \geq\|A \psi-\langle A\rangle \psi\|=\sigma(A)
$$

for any real number $\lambda$, where $\langle A\rangle=(\psi, A \psi)$, (AR) implies the following statement: If an observable $A$ in a system $\mathbf{S}$ is measured with mean error $\varepsilon(A)$, then the post-measurement standard deviation $\sigma(A)$ of A satisfies

$$
\sigma(A) \leq \varepsilon(A) .
$$

\section{HEISENBERG'S DERIVATION OF THE UNCERTAINTY PRINCIPLE}

Heisenberg's derivation of (1) starts with considering a state $\psi$ just after the measurement of the position observable $\hat{q}$ to obtain the outcome $q^{\prime}$ with mean error $\varepsilon(\hat{q})$ and consider what relation holds between $\varepsilon(\hat{q})$ and $\varepsilon(\hat{p})$ if the momentum observable $\hat{p}$ has been measured simultaneously to obtain the outcome $p^{\prime}$ with mean error $\varepsilon(\hat{p})$. Then, by (AR) or Eq. (8) the state $\psi$ should have the position standard deviation $\sigma(\hat{q})$ satsifying

$$
\sigma(\hat{q}) \leq \varepsilon(\hat{q})
$$

Heisenberg actually supposed that the state $\psi$ is a Gaussian wave function $\frac{1}{-}$ (p. 69)

$$
\psi(q)=\frac{1}{\left(\pi q_{1}^{2}\right)^{1 / 4}} \exp \left[-\frac{\left(q-q^{\prime}\right)^{2}}{2 q_{1}^{2}}-\frac{i}{\hbar} p^{\prime}\left(q-q^{\prime}\right)\right],
$$

which is later known as a minimum uncertainty wave packet, with its Fourier transform

$$
\hat{\psi}(p)=\frac{1}{\left(\pi p_{1}^{2}\right)^{1 / 4}} \exp \left[-\frac{\left(p-p^{\prime}\right)^{2}}{2 p_{1}^{2}}+\frac{i}{\hbar} q^{\prime}\left(p-p^{\prime}\right)\right],
$$

and he proved relation (2) for the state $\psi$ given by Eq. (10).

Exactly this part of Heisenberg's argument was generalized by Kennard ${ }^{2}$ to prove relation (3) for any vector state $\psi$. Thus, Kennard $^{2}$ relaxed Heisenberg's assumption on the state $\psi$ to the assumption that the state $\psi$ after the position measurement can be arbitrary wave function $\psi$ satisfying $E q$. (9). Then, if the momentum observable $\hat{p}$ has been measured simultaneously to obtain the outcome $p^{\prime}$ with an error $\varepsilon(\hat{p})$, by (AR) or Eq. (8) again the state $\psi$ should also satisfy the relation

$$
\sigma(\hat{p}) \leq \varepsilon(\hat{p}) .
$$

Therefore, Heisenberg's uncertainty relation (4) immediately follows from Kennard's relation (3).

As above Heisenberg in 1927 not only derived relation (1) by the $\gamma$-ray thought experiment but also gave its mathematical proof. However, he supposed the repeatability hypothesis or its approximate version as an additional but obsolete assumption in addition to the standard postulates for quantum mechanics.

The approximate repeatability hypothesis (AR) has not been explicitly formulated in the literature, but in the following explanation on the derivation of the uncertainty principle von Neumann $\underline{\underline{3}}$ (pp. 238-239) assumed (AR):

We are then to show that if $Q, P$ are two canonically conjugate quantities, and a system is in a state in which the value of $Q$ can be given with the accuracy $\varepsilon$ (i.e., by a $Q$ measurement with an error range $\varepsilon$ ), then $P$ can be known with no greater accuracy than $\eta=\hbar /(2 \varepsilon)$. Or: a measurement of $Q$ with the accuracy $\varepsilon$ must bring about an indeterminacy $\eta=\hbar /(2 \varepsilon)$ in the value of $P$.

In the above, it is obviously assumed that a state with the position standard deviation $\varepsilon$ is resulted by a $Q$ measurement with an error range $\varepsilon$. This assumption is what we have generally formulated in Eq. (8) as an immediate logical consequence of (AR).

Two inequalities (3) and (4) are often distinguished as the preparational uncertainty relation and the measuremental uncertainty relation, respectively. However, under the repeatability hypothesis such a distinction is not apparent, since a measurement is required to prepare the state with a sharp value of the measured observable. In fact, the above argument shows that there exists an immediate logical relationship between those two inequalities.

\section{ABANDONING THE REPEATABILITY HYPOTHESIS}

The repeatability hypothesis explains only a restricted class of measurements and does not generally characterize the state changes caused by quantum measurements. In fact, there exist commonly used measurements of discrete observables, such as photon counting, that do not satisfy the repeatability hypothesis 15 . Moreover, it has been shown that the repeatability hypothesis cannot be generalized to continuous observables in the standard formulation of quantum mechanics $16-19$. In 1970, Davies and Lewis ${ }^{20}$ proposed abandoning the repeatability hypothesis and introduced a new mathematical framework to treat all the physically realizable quantum measurements:

One of the crucial notions is that of repeatability which we show is implicitly assumed in most of the axiomatic treatments of quantum mechanics, but whose abandonment leads to a much more flexible approach to measurement theory $\underline{20}$ (p. 239).

Denote by $\tau c(\mathscr{H})$ the space of trace class operators on $\mathscr{H}$, by $\mathscr{S}(\mathscr{H})$ the space of density operators on $\mathscr{H}$, and by $P(\tau c(\mathscr{H}))$ the space of positive linear maps on $\tau c(\mathscr{H})$. Davies and Lewis ${ }^{20}$ introduced a mathematical notion of instrument as follows. A Davies-Lewis (DL) instrument for (a system $\mathbf{S}$ described by) a Hilbert space $\mathscr{H}$ is defined as 
a $P(\tau c(\mathscr{H}))$-valued Borel measure $\mathscr{I}$ on $\mathbb{R}$ countably additive in the strong operator topology such that $\mathscr{I}(\mathbb{R})$ is tracepreserving $(\operatorname{Tr}[\mathscr{I}(\mathbb{R}) \rho]=\operatorname{Tr}[\rho])$.

Let $\mathbf{A}(\mathbf{x})$ be a measuring apparatus for $\mathbf{S}$ with the output variable $\mathbf{x}$. The statistical properties of the apparatus $\mathbf{A}(\mathbf{x})$ are determined by (i) the probability distribution $\operatorname{Pr}\{\mathbf{x} \in \Delta \| \rho\}$ of the outcome $\mathbf{x}$ in an arbitrary state $\rho$, and (ii) the state change $\rho \rightarrow \rho_{\{\mathbf{x} \in \Delta\}}$ from the state $\rho$ just before the measurement to the state $\rho_{\{\mathbf{x} \in \Delta\}}$ just after the measurement given the condition $\mathbf{x} \in \Delta$. The proposal of Davies and Lewis ${ }^{20}$ can be stated as follows.

(DL) The Davies-Lewis thesis. For every measuring apparatus $\mathbf{A}(\mathbf{x})$ with output variable $\mathbf{x}$ there exists a unique $D L$ instrument $\mathscr{I}$ satisfying

$$
\begin{aligned}
\operatorname{Pr}\{\mathbf{x} \in \Delta \| \rho\} & =\operatorname{Tr}[\mathscr{I}(\Delta) \rho], \\
\rho \rightarrow \rho_{\{\mathbf{x} \in \Delta\}} & =\frac{\mathscr{I}(\Delta) \rho}{\operatorname{Tr}[\mathscr{I}(\Delta) \rho]} .
\end{aligned}
$$

For any $\Delta \in \mathscr{B}(\mathbb{R})$, define $\Pi(\Delta)$ by

$$
\Pi(\Delta)=\mathscr{I}(\Delta)^{*} 1
$$

where $\mathscr{I}(\Delta)^{*}$ is the dual map of $\mathscr{I}(\Delta)$ given by $\operatorname{Tr}\left[\left(\mathscr{I}(\Delta)^{*} X\right) \rho\right]=\operatorname{Tr}[X(\mathscr{I}(\Delta) \rho)]$ for all $X \in \mathscr{L}(\mathscr{H})$. Then, the map $\Delta \rightarrow \Pi(\Delta)$ is a probability operator-valued measure $(\mathrm{POVM})^{21}$, called the POVM of $\mathscr{I}$, satisfying

$$
\operatorname{Pr}\{\mathbf{x} \in \Delta \| \rho\}=\operatorname{Tr}[\Pi(\Delta) \rho]
$$

for all $\rho \in \mathscr{S}(\mathscr{H})$ and $\Delta \in \mathscr{B}(\mathbb{R})$.

The problem of mathematically characterizing all the physically realizable quantum measurements is reduced to the problem as to which instruments are physically realizable ${ }^{13}$. To settle this problem, standard models of measuring processes were introduced in ${ }^{16}$ as follows. A measuring process for (a system described by) a Hilbert space $\mathscr{H}$ is defined as a quadruple $\left(\mathscr{K}, \rho_{0}, U, M\right)$ consisting of a Hilbert space $\mathscr{K}$, a density operator $\rho_{0}$ on $\mathscr{K}$, a unitary operator $U$ on $\mathscr{H} \otimes \mathscr{K}$, and a self-adjoint operator $M$ on $\mathscr{K}$. A measuring process $\left(\mathscr{K}, \rho_{0}, U, M\right)$ is said to be pure if $\rho_{0}$ is a pure state, and it is said to be separable if $\mathscr{K}$ is separable.

The measuring process $\left(\mathscr{K}, \rho_{0}, U, M\right)$ mathematically models the following description of a measurement. The measurement is carried out by the interaction, referred to as the measuring interaction, between the object $\mathbf{S}$ and the probe $\mathbf{P}$. The probe $\mathbf{P}$ is described by the Hilbert space $\mathscr{K}$ and prepared in the state $\rho_{0}$ just before the measurement. The time evolution of the composite system $\mathbf{P}+\mathbf{S}$ during the measuring interaction is described by the unitary operator $U$. The outcome of the measurement is obtained by measuring the observable $M$ called the meter observable of the probe $\mathbf{P}$ just after the measuring interaction. We assume that the measuring interaction turns on at time $t=0$ and turns off at time $t=\Delta t$. In the Heisenberg picture, we write

$$
A_{1}(0)=A_{1} \otimes 1, \quad A_{2}(0)=1 \otimes A_{2}, \quad A_{12}(\Delta t)=U^{\dagger} A_{12}(0) U,
$$

for an observable $A_{1}$ of $\mathbf{S}$, an observable $A_{2}$ of $\mathbf{P}$, and an observable $A_{12}(0)$ of $\mathbf{S}+\mathbf{P}$.

Suppose that the measurement carried out by an apparatus $\mathbf{A}(\mathbf{x})$ is described by a measuring process $\left(\mathscr{K}, \rho_{0}, U, M\right)$. Then, it is shown ${ }^{16}$ that the statistical properties of the apparatus $\mathbf{A}(\mathbf{x})$ is given by

$$
\begin{aligned}
\operatorname{Pr}\{\mathbf{x} \in \Delta \| \rho\} & =\operatorname{Tr}\left[E^{M(\Delta t)}(\Delta)\left(\rho \otimes \rho_{0}\right)\right], \\
\rho \rightarrow \rho_{\{\mathbf{x} \in \Delta\}} & =\frac{\operatorname{Tr}_{\mathscr{K}}\left[\left(1 \otimes E^{M}(\Delta)\right) U\left(\rho \otimes \rho_{0}\right) U^{\dagger}\right]}{\operatorname{Tr}\left[E^{M(\Delta t)}(\Delta)\left(\rho \otimes \rho_{0}\right)\right]},
\end{aligned}
$$

where $\operatorname{Tr}_{\mathscr{K}}$ stands for the partial trace on the Hilbert space $\mathscr{K}$. The POVM $\Pi$ of the apparatus $\mathbf{A}(\mathbf{x})$ is defined by

$$
\Pi(\Delta)=\operatorname{Tr}_{\mathscr{K}}\left[E^{M(\Delta t)}(\Delta)\left(1 \otimes \rho_{0}\right)\right]
$$

for any $\Delta \in \mathscr{B}(\mathbb{R})$. Then, the map $\Delta \rightarrow \Pi(\Delta)$ is a probability operator-valued measure (POVM) ${ }^{21}$ satisfying

$$
\operatorname{Pr}\{\mathbf{x} \in \Delta \| \rho\}=\operatorname{Tr}[\Pi(\Delta) \rho]
$$

for all $\rho \in \mathscr{S}(\mathscr{H})$ and $\Delta \in \mathscr{B}(\mathbb{R})$.

Now it is easy to see that the above description of the measurement statistics of the apparatus $\mathbf{A}(\mathbf{x})$ is consistent with the Davies-Lewis thesis. In fact, the relation

$$
\mathscr{I}(\Delta) \rho=\operatorname{Tr}_{\mathscr{K}}\left[\left(1 \otimes E^{M}(\Delta)\right) U\left(\rho \otimes \rho_{0}\right) U^{\dagger}\right]
$$

defines a DL instrument $\mathscr{I}$. In this case, we say that the instrument $\mathscr{I}$ is realized by the measuring process $\left(\mathscr{K}, \rho_{0}, U, M\right)$.

A DL instrument for $\mathscr{H}$ is said to be completely positive $(C P)$ if $\mathscr{I}(\Delta)$ is completely positive for every $\Delta \in \mathscr{B}(\mathbb{R})$, i.e., $\mathscr{I}(\Delta) \otimes \mathrm{id}_{n}: \tau c(\mathscr{H}) \otimes M_{n} \rightarrow \tau c(\mathscr{H}) \otimes M_{n}$ is a positive map for every finite number $n$, where $M_{n}$ is the matrix algebra of order $n$ and $\mathrm{id}_{n}$ is the identity map on $M_{n}$. The following theorem characterizes the physically realizable DL instruments by completely positivity 16,22 .

Theorem 1 (Realization theorem for CP instruments). $A$ $D L$ instrument can be realized by a measuring process if and only if it is completely positive. In particular, every CP instrument can be realized by a pure measuring process, and if $\mathscr{H}$ is separable, every CP instrument for $\mathscr{H}$ can be realized by a pure and separable measuring process.

Now, we have reached the following general measurement axiom, abandoning Axiom 4 or the repeatability hypothesis.

Axiom 5 (General measurement axiom). To every measuring apparatus $\mathbf{A}(\mathbf{x})$ with output variable $\mathbf{x}$ there exists a unique CP instrument $\mathscr{I}$ satisfying Eqs. (13) and (14). Conversely, to every instrument $\mathscr{I}$ there exists at least one measuring apparatus $\mathbf{A}(\mathbf{x})$ satisfying Eqs. (13) and (14).

\section{VON NEUMANN'S MODEL OF POSITION MEASUREMENT}

Let $A$ and $B$ be observables of a system $\mathbf{S}$ described by a Hilbert space $\mathscr{H}$. Let $\mathbf{A}(\mathbf{x})$ be a measuring apparatus for $\mathbf{S}$ 
with the output variable $\mathbf{x}$ described by a measuring process $\mathbf{M}=\left(\mathscr{K}, \rho_{0}, U, M\right)$ from time $t=0$ to $t=\Delta t$. An approximate simultaneous measurement of $A(0)$ and $B(0)$ is obtained by direct simultaneous measurement of commuting observables $M(\Delta t)$ and $B(\Delta t)$, where $M(\Delta t)$ is considered to approximately measure $A(0)$ and $B(\Delta t)$ is considered to approximately measure $B(0)$. In this case the error of the $B(0)$ measurement is called the disturbance of $B$ caused by the measuring process $\mathbf{M}$, and the relation for the errors of the $A(0)$ measurement and the $B(0)$ measurement is called the errordisturbance relation $(E D R)$. In what follows, we examine the EDR for position measurement error and momentum disturbance.

Until 1980's only solvable model of position measurement had been given by von Neumann ${ }^{3}$. We show that this long-standing model satisfies Heisenberg's error-disturbance relation ${ }^{11}$, a version of Heisenberg's uncertainty relation (4).

Consider a one-dimensional mass $\mathbf{S}$, called an object, with position $\hat{x}$ and momentum $\hat{p}_{x}$, described by a Hilbert space $\mathscr{H}=L^{2}\left(\mathbb{R}_{x}\right)$, where $\mathbb{R}_{x}$ is a copy of the real line. The object is coupled from time $t=0$ to $t=\Delta t$ with the probe $\mathbf{P}$, another one-dimensional mass with position $\hat{y}$ and momentum $\hat{p}_{y}$, described by a Hilbert space $\mathscr{K}=L^{2}\left(\mathbb{R}_{y}\right)$, where $\mathbb{R}_{y}$ is another copy of the real line. The outcome of the measurement is obtained by measuring the probe position $\hat{y}$ at time $t=\Delta t$. The total Hamiltonian for the object and the probe is taken to be

$$
H_{\mathbf{S}+\mathbf{P}}=H_{\mathbf{S}}+H_{\mathbf{P}}+K H,
$$

where $H_{\mathbf{S}}$ and $H_{\mathbf{P}}$ are the free Hamiltonians of $\mathbf{S}$ and $\mathbf{P}$, respectively, $H$ represents the measuring interaction. The coupling constant $K$ satisfies $K \Delta t=1$ and it is so strong $(K \gg 1)$ that $H_{\mathbf{S}}$ and $H_{\mathbf{P}}$ can be neglected.

The measuring interaction $H$ is given by

$$
H=\hat{x} \otimes \hat{p}_{y},
$$

so that the unitary operator of the time evolution of $\mathbf{S}+\mathbf{P}$ from $t=0$ to $t=\tau \leq \Delta t$ is given by

$$
U(\tau)=\exp \left(\frac{-i K \tau}{\hbar} \hat{x} \otimes \hat{p}_{y}\right)
$$

Suppose that the object $\mathbf{S}$ and the probe $\mathbf{P}$ are in the vector states $\psi$ and $\xi$, respectively, just before the measurement. We assume that the wave functions $\psi(x)$ and $\xi(y)$ are Schwartz rapidly decreasing functions $\mathrm{s}^{23}$. Then, the time evolution of $\mathbf{S}+\mathbf{P}$ in the time interval $(0, \Delta t)$ is given by the unitary operator $U(\Delta t)=e^{-i \hat{x} \otimes \hat{p}_{y} / \hbar}$. Thus, this measuring process is represented by $\left(L^{2}\left(\mathbb{R}_{y}\right),|\xi\rangle\langle\xi|, e^{-i \hat{x} \otimes \hat{p}_{y} / \hbar}, \hat{y}\right)$.

The state of the composite system $\mathbf{S}+\mathbf{P}$ just after the measurement is $U(\Delta t) \psi \otimes \xi$. By solving the Schrödinger equation, we have

$$
U(\Delta t)(\psi \otimes \xi)(x, y)=\psi(x) \xi(y-x) .
$$

From this, the probability distribution of output variable $\mathbf{x}$ is given by

$$
\operatorname{Pr}\{\mathbf{x} \in \Delta \| \psi\}=\int_{\Delta} d y \int_{\mathbb{R}}|\psi(x)|^{2}|\xi(y-x)|^{2} d x .
$$

By a property of convolution, if the probe probability distribution $|\xi(y)|^{2}$ approaches to the Dirac delta function $\delta(y)$, the output probability approaches to the Born probability distribution $|\psi(x)|^{2}$.

The corresponding instrument $\mathscr{I}$ is given by

$$
\mathscr{I}(\Delta) \rho=\int_{\Delta} \xi(y 1-\hat{x}) \rho \xi(y 1-\hat{x})^{\dagger} d y,
$$

and the corresponding POVM is given by

$$
\Pi(\Delta)=\int_{\Delta}|\xi(y 1-\hat{x})|^{2} d y,
$$

Solving the Heisenberg equations of motion, we have

$$
\begin{aligned}
\hat{x}(\Delta t) & =\hat{x}(0), \\
\hat{y}(\Delta t) & =\hat{x}(0)+\hat{y}(0), \\
\hat{p}_{x}(\Delta t) & =\hat{p}_{x}(0)-\hat{p}_{y}(0), \\
\hat{p}_{y}(\Delta t) & =\hat{p}_{y}(0) .
\end{aligned}
$$

\section{ROOT-MEAN-SQUARE ERROR AND DISTURBANCE}

To define the "mean error" of the above position measurement, let us recall classical definitions. Suppose that a quantity $X=x$ is measured by directly observing another quantity $Y=y$. For each pair of values $(X, Y)=(x, y)$, the error is defined as $y-x$. To define the "mean error" given the joint probability distribution (JPD) $\mu^{X, Y}(d x, d y)$ of $X$ and $Y$, Gauss ${ }^{24}$ introduced the root-mean-square (rms) error $\varepsilon_{G}(X, Y)$ of $Y$ for $X$ as

$$
\varepsilon_{G}(X, Y)=\left(\iint_{\mathbb{R}^{2}}(y-x)^{2} \mu^{X, Y}(d x, d y)\right)^{1 / 2},
$$

which Gauss 24 called the "mean error" or the "mean error to be feared", and has long been accepted as a standard definition for the "mean error".

In the von Neumann model, the observable $\hat{x}(0)$ is measured by directly observing the meter observable $\hat{y}(\Delta t)$. Since $\hat{x}(0)$ and $\hat{y}(\Delta t)$ commute by Eq. (30), we have the JPD $\mu^{\hat{x}(0), \hat{y}(\Delta t)}(d x, d y)$ of $\hat{x}(0)$ and $\hat{y}(\Delta t)$ as

$$
\mu^{\hat{x}(0), \hat{y}(\Delta t)}(d x, d y)=\left\langle E^{\hat{x}(0)}(d x) E^{\hat{y}(\Delta t)}(d y)\right\rangle,
$$

where $\langle\cdots\rangle$ stands for the mean value in the state $\psi \otimes \xi$. Then, by Eq. (33) the rms error $\varepsilon(\hat{x}, \psi)$ for measuring $\hat{x}$ in state $\psi$ is defined as the rms error $\varepsilon_{G}(\hat{x}(0), \hat{y}(\Delta t))$ of $\hat{y}(\Delta t)$ for $\hat{x}(0)$, so that we have

$$
\begin{aligned}
\varepsilon(\hat{x}, \psi) & =\left(\iint_{\mathbb{R}^{2}}(y-x)^{2} \mu^{\hat{x}(0), \hat{y}(\Delta t)}(d x, d y)\right)^{1 / 2} \\
& =\left\langle(\hat{y}(\Delta t)-\hat{x}(0))^{2}\right\rangle^{1 / 2}=\left\langle\hat{y}(0)^{2}\right\rangle^{1 / 2} .
\end{aligned}
$$

Since $\hat{p}_{x}(0)$ and $\hat{p}_{x}(\Delta t)$ also commute from Eq. 31), we also have the JPD $\mu^{\hat{p}_{x}(0), \hat{p}_{x}(\Delta t)}(d x, d y)$ of the values of $\hat{p}_{x}(0)$ and $\hat{p}_{x}(\Delta t)$. The rms disturbance $\eta\left(\hat{p}_{x}, \psi\right)$ of $\hat{p}_{x}$ in state $\psi$ is 
similarly defined as the rms error $\varepsilon_{G}\left(\hat{p}_{x}(0), \hat{p}_{x}(\Delta)\right)$, so that we have

$$
\begin{aligned}
\eta\left(\hat{p}_{x}, \psi\right) & =\left(\iint_{\mathbb{R}^{2}}(y-x)^{2} \mu^{\hat{p}_{x}(0), \hat{p}_{x}(\Delta t)}(d x, d y)\right)^{1 / 2} \\
& =\left\langle\left(\hat{p}_{x}(\Delta t)-\hat{p}_{x}(0)\right)^{2}\right\rangle^{1 / 2}=\left\langle\hat{p}_{y}(0)^{2}\right\rangle^{1 / 2} .
\end{aligned}
$$

Then, by Kennard's inequality (3) we have

$$
\begin{aligned}
\varepsilon(\hat{x}, \psi) \eta\left(\hat{p}_{x}, \psi\right) & =\left\langle\hat{y}(0)^{2}\right\rangle^{1 / 2}\left\langle\hat{p}_{y}(0)^{2}\right\rangle^{1 / 2} \\
& \geq \sigma(\hat{y}(0)) \sigma\left(\hat{p}_{y}(0)\right) \geq \frac{\hbar}{2} .
\end{aligned}
$$

Thus, the von Neumann model satisfies Heisenberg's errordisturbance relation (EDR)

$$
\varepsilon(\hat{x}) \eta\left(\hat{p}_{x}\right) \geq \frac{\hbar}{2}
$$

for $\varepsilon(\hat{x})=\varepsilon(\hat{x}, \psi)$ and $\eta\left(\hat{p}_{x}\right)=\eta\left(\hat{p}_{x}, \psi\right)$.

By the limited availability for measurement models up to the 1980's, the above result appears to have enforced a prevailing belief in Heisenberg's EDR (38), for instance, in claiming the standard quantum limit for gravitational wave detection $25-27$.

\section{MEASUREMENT VIOLATING HEISENBERG'S EDR}

In 1980, Braginsky, Vorontsov, and Thorne ${ }^{25}$ claimed that Heisenberg's EDR (38) leads to a sensitivity limit, called the standard quantum limit (SQL), for gravitational wave detectors exploiting free-mass position monitoring. Subsequently, Yuen ${ }^{28}$ questioned the validity of the SQL, and then Caves ${ }^{27}$ defended the SQL by giving a new formulation and a new proof without directly appealing to Heisenberg's ERD 38. Eventually, the conflict was reconciled 29.30 by pointing out that Caves 27 still supposed (AR), in spite of avoiding Heisenberg's ERD (38). More decisively, a solvable model of a precise position measurement was also constructed that breaks the SQL 29.30 ; later this model was shown to break Heisenberg's EDR 38$]^{31}$.

In what follows, we examine this model, which modifies the measuring interaction of the von Neumann model. In this new model, the object, the probe, and the probe observables, the coupling constant $K$, and the time duration $\Delta t$ are the same as the von Neumann model. The measuring interaction is taken to be 29

$$
H=\frac{\pi}{3 \sqrt{3}}\left(2 \hat{x} \otimes \hat{p}_{y}-2 \hat{p}_{x} \otimes \hat{y}+\hat{x} \hat{p}_{x} \otimes 1-1 \otimes \hat{y} \hat{p}_{y}\right) .
$$

The corresponding instrument is give by $\underline{\underline{13}}$

$$
\mathscr{I}(\Delta) \rho=\int_{\Delta} e^{-i x \hat{p}_{x}}|\phi\rangle\langle\phi| e^{-i x \hat{p}_{x}} \operatorname{Tr}\left[E^{\hat{x}}(d x) \rho\right],
$$

where $\phi(x)=\xi(-x)$, and the corresponding POVM is given by

$$
\Pi(\Delta)=E^{A}(\Delta) .
$$

Solving the Heisenberg equations of motion, we have

$$
\begin{aligned}
\hat{x}(\Delta t) & =\hat{x}(0)-\hat{y}(0), \\
\hat{y}(\Delta t) & =\hat{x}(0), \\
\hat{p}_{x}(\Delta t) & =-\hat{p}_{y}(0), \\
\hat{p}_{y}(\Delta t) & =\hat{p}_{x}(0)+\hat{p}_{y}(0) .
\end{aligned}
$$

Thus, $\hat{x}(0)$ and $\hat{y}(\Delta t)$ commute and also $\hat{p}_{x}(0)$ and $\hat{p}_{x}(\Delta t)$ commute, so that the rms error and the rms disturbance are well defined by their JPDs, and given by

$$
\begin{aligned}
\varepsilon(\hat{x}, \psi) & =0, \\
\eta\left(\hat{p}_{x}, \psi\right) & =\left\langle\left(\hat{p}_{y}(0)+\hat{p}_{x}(0)\right)^{2}\right\rangle^{1 / 2}<\infty .
\end{aligned}
$$

Consequently, we have

$$
\varepsilon(\hat{x}) \eta\left(\hat{p}_{x}\right)=0 .
$$

Therefore, this model obviously violates Heisenberg's EDR (38).

\section{UNIVERSALLY VALID ERROR-DISTURBANCE RELATION}

To derive a universally valid EDR, consider a measuring process $\mathbf{M}=\left(\mathscr{K}, \rho_{0}, U, M\right)$. If $A(0)$ and $M(\Delta t)$ commute, the rms error of the measuring process $\mathbf{M}$ for measuring $A$ in $\rho$ can be defined through the JPD of $A(0)$ and $M(\Delta t)$. Similarly, if $B(0)$ and $B(\Delta t)$ commute, the rms disturbance can also be defined through the JPD of $B(0)$ and $B(\Delta t)$. In order to extend the definitions of the rms error and disturbance to the general case, we introduce the noise operator and the disturbance operator.

The noise operator $N(A)$ is defined as the difference $M(\Delta t)-A(0)$ between the observable $A(0)$ to be measured and the meter observable $M(\Delta t)$ to be read and the disturbance operator $D(A)$ is defined as the the change $B(\Delta t)-B(0)$ of $B$ caused by the measuring interaction, i.e.,

$$
\begin{aligned}
& N(A)=M(\Delta t)-A(0), \\
& D(B)=B(\Delta t)-B(0) .
\end{aligned}
$$

The mean noise operator $n(A)$ and the mean disturbance operator $d(B)$ are defined by

$$
\begin{aligned}
n(A) & =\operatorname{Tr}_{\mathscr{K}}\left[N(A) 1 \otimes \rho_{0}\right], \\
d(B) & =\operatorname{Tr}_{\mathscr{K}}\left[D(B) 1 \otimes \rho_{0}\right] .
\end{aligned}
$$

The rms error $\varepsilon(A, \rho)$ and the rms disturbance $\eta(B, \rho)$ for observables $A, B$, respectively, in state $\rho$ are defined by

$$
\begin{aligned}
\varepsilon(A, \rho) & =\left(\operatorname{Tr}\left[N(A)^{2} \rho \otimes \rho_{0}\right]\right)^{1 / 2}, \\
\eta(B, \rho) & =\left(\operatorname{Tr}\left[D(B)^{2} \rho \otimes \rho_{0}\right]\right)^{1 / 2} .
\end{aligned}
$$

An immediate meaning of $\varepsilon(A, \rho)$ and $\eta(B, \rho)$ are the rms's of the noise operator and the disturbance operator.

Suppose that $M(\Delta t)$ and $A(0)$ commute in $\rho \otimes \rho_{0}$, i.e.,

$$
\left[E^{A(0)}(\Delta), E^{M(\Delta t)}(\Gamma)\right] \rho \otimes \rho_{0}=0
$$


for all $\Delta, \Gamma \in \mathscr{B}(\mathbb{R})^{32-34}$. In this case, the relation

$$
\mu^{A(0), M(\Delta t)}(d x, d y)=\operatorname{Tr}\left[E^{A(0)}(d x) E^{M(\Delta t)}(d y) \rho \otimes \rho_{0}\right]
$$

defines the JPD of $A(0)$ and $M(\Delta t)$ satisfying

$$
\operatorname{Tr}\left[p(A(0), M(\Delta t)) \rho \otimes \rho_{0}\right]=\iint_{\mathbb{R}^{2}} p(x, y) \mu^{A(0), M(\Delta t)}(d x, d y)
$$

for any real polynomial $p(A(0), M(\Delta t))$ in $A(0)$ and $M(\Delta t)^{32}$. Thus, the classical rms error $\varepsilon_{G}(A(0), M(\Delta t))$ of $M(\Delta t)$ for $A(0)$ is well defined, and we easily obtain the relation

$$
\varepsilon(A, \rho)=\varepsilon_{G}(A(0), M(\Delta t)) .
$$

Similarly, we have $\eta(B, \rho)=\varepsilon_{G}(B(0), B(\Delta t))$ if $B(0)$ and $B(\Delta t)$ commute in $\rho \otimes \rho_{0}$.

In 2003 , the present author ${ }^{11,12,35}$ derived the relation

$$
\varepsilon(A) \eta(B)+|\langle[n(A), B]\rangle+\langle[A, d(B)]\rangle| \geq \frac{1}{2}|\langle[A, B]\rangle|,
$$

where $\varepsilon(A)=\varepsilon(A, \rho), \eta(B)=\eta(B, \rho)$, which is universally valid for any observables $A, B$, any system state $\rho$, and any measuring process M. From Eq. [59), it is concluded that if the error and the disturbance are statistically independent from system state, then the Heisenberg type EDR

$$
\varepsilon(A) \eta(B) \geq \frac{1}{2}|\langle[A, B]\rangle|
$$

holds, extending the previous results $36-39$. The additional correlation term in Eq. (59) allows the error-disturbance product $\varepsilon(A) \eta(B)$ to violate the Heisenberg type EDR 60 . In general, the relation

$$
\varepsilon(A) \eta(B)+\varepsilon(A) \sigma(B)+\sigma(A) \eta(B) \geq \frac{1}{2}|\langle[A, B]\rangle|,
$$

holds for any observables $A, B$, any system state $\rho$, and any measuring process $\mathbf{M}^{11}-13,35,40,41$.

The new relation (61) leads to the following new constraints for precise measurements and non-disturbing measurement: then

$$
\begin{aligned}
\sigma(A) \eta(B) \geq \frac{1}{2}|\langle[A, B]\rangle|, & \text { if } \varepsilon(A)=0, \\
\varepsilon(A) \sigma(B) \geq \frac{1}{2}|\langle[A, B]\rangle|, & \text { if } \eta(B)=0 .
\end{aligned}
$$

Note that if $\langle[A, B]\rangle \neq 0$, the Heisenberg type EDR (60) leads to the divergence of $\varepsilon(A)$ or $\eta(B)$ in those cases. The new error bound Eq. (63) was used to derive a conservationlaw-induced limits for measurements ${ }^{12,42-44}$ quantitatively generalizing the Wigner-Araki-Yanase theorem ${ }^{45}-48$ and was used to derive a fundamental accuracy limit for quantum computing $\frac{12}{}$.

\section{QUANTUM ROOT MEAN SQUARE ERRORS}

We say that the measuring process $\mathbf{M}$ is probability reproducible for the observable $A$ in the state $\rho$ iff

$$
\operatorname{Tr}\left[E^{M(\Delta t)}(\Delta) \rho \otimes \rho_{0}\right]=\operatorname{Tr}\left[E^{A}(\Delta) \rho\right]
$$

holds for all $\Delta \in \mathscr{B}(\mathbb{R})$. The rms error $\varepsilon(A, \rho)$ satisfies that $\rho(A, \rho)=0$ for all $\rho$ if and only if $\mathbf{M}$ is probability reproducible for $A$ in all $\rho \frac{13,31}{2}$. Thus, the condition that $\varepsilon(A, \rho)=0$ for all $\rho$ characterizes the class of measurements with POVM $\Pi$ satisfying $\Pi=E^{A}$.

Busch, Heinonen, and Lahti $\frac{49}{}$ pointed out that there are cases where $\varepsilon(A, \rho)=0$ holds but $\mathbf{M}$ is not probability reproducible and where $\mathbf{M}$ is not probability reproducible but $\varepsilon(A, \rho)=0$ holds, and questioned the reliability of the rms error $\varepsilon(A . \rho)$ as a state-dependent error measure. However, their argument lacks a reasonable definition of precise measurements, necessary for discussing the reliability of error measures. In response to their criticism, the present author ${ }^{33,34}$ has successfully characterized the precise measurements of $A$ in a given state $\rho$ and shown that the rms error $\varepsilon(A, \rho)$ reliably characterizes such measurements. In what follows we survey those results, which were mostly neglected in the recent debates $50-52$.

Let us start with the classical case. Suppose that a quantity $X=x$ is measured by direct observation of another quantity $Y=y$. Then, this measurement is precise iff $X=Y$ holds with probability 1 , or equivalently the JPD $\mu^{X, Y}(d x, d y)$ of $X$ and $Y$ concentrates on the diagonal set, i.e.,

$$
\mu^{X, Y}\left(\left\{(x, y) \in \mathbb{R}^{2} \mid x \neq y\right\}\right)=0 .
$$

As easily seen from Eq. (33), this condition is equivalent to the condition $\varepsilon_{G}(X, Y)=0$.

Generalizing the classical case, we say that a measuring process $\mathbf{M}$ makes a strongly precise measurement of $A$ in $\rho$ iff $A(0)=M(\Delta t)$ holds with probability 1 in the sense that $A(0)$ and $M(\Delta t)$ commute in $\rho \otimes \rho_{0}$ and that the JPD $\mu^{A(0), M(\Delta t)}$ concentrates on the diagonal set, i.e.,

$$
\mu^{A(0), M(\Delta t)}\left(\left\{(x, y) \in \mathbb{R}^{2} \mid x \neq y\right\}\right)=0 .
$$

On the other hand, we have introduced another operational requirement. The weak joint distribution $\mu_{W}^{A(0), M(\Delta t)}$ of $A(0)$ and $M(\Delta t)$ in a state $\rho$ is defined by

$$
\mu_{W}^{A(0), M(\Delta t)}(d x, d y)=\operatorname{Tr}\left[E^{A(0)}(d x) E^{M(\Delta t)}(d y) \rho \otimes \rho_{0}\right] .
$$

The weak joint distribution is not necessarily positive but operationally accessible by weak measurement and postselection $\underline{53}$. We say that the measuring process $\mathbf{M}$ makes a weakly precise measurement of $A$ in $\rho$ iff the weak joint distribution $\mu_{W}^{A(0), M(\Delta t)}$ in state $\rho$ concentrates on the diagonal set, i.e.,

$$
\mu_{W}^{A(0), M(\Delta t)}\left(\left\{(x, y) \in \mathbb{R}^{2} \mid x \neq y\right\}\right)=0 .
$$

This condition does not require that $A(0)$ and $M(\Delta t)$ commute, while it only requires that the weak joint distribution concentrates on the event $A(0)=M(\Delta t)$. A similar condition has been used to observe momentum transfer in a doubleslit 'which-way' experiment ${ }^{54.55}$. We naturally consider that strongly preciseness is a sufficient condition for precise measurements and weak preciseness is a necessary condition. In the previous investigations ${ }^{33,34}$, it was mathematically proved 
that both conditions are equivalent. Thus, either condition is concluded to be a necessary and sufficient condition characterizing the unique class of precise measurements. As above, we say that the measuring process $\mathbf{M}$ precisely measures $A$ in $\rho$ iff it makes a strongly or weakly precise measurement of $A$ in $\rho$.

To characterize the class of precise measurements in terms of the rms error-freeness condition, $\varepsilon(A, \rho)=0$, and the probability reproducibility condition, we introduce the following notions. The cyclic subspace $\mathscr{C}(A, \rho)$ generated by $A$ and $\rho$ is defined as the closed subspace of $\mathscr{H}$ generated by $\left\{E^{A}(\Delta) \phi \mid \Delta \in \mathscr{B}(\mathbb{R}), \phi \in \operatorname{ran}(\rho)\right\}$, where $\operatorname{ran}(\rho)$ denotes the range of $\rho$. Then, the following theorem holds 33,34 .

Theorem 2. Let $\mathbf{M}=\left(\mathscr{K}, \rho_{0}, U, M\right)$ be a measuring process for the system $\mathbf{S}$ described by a Hilbert space $\mathscr{H}$. Let $A$ be an observable of $\mathbf{S}$ and $\rho$ a state of $\mathbf{S}$. Then, the following conditions are equivalent.

(i) $\mathbf{M}$ precisely measures $A$ in $\rho$.

(ii) $\varepsilon(A, \phi)=0$ in all $\phi \in \mathscr{C}(A, \rho)$.

(iii) $\mathbf{M}$ is probability reproducible for $A$ in all $\phi \in \mathscr{C}(A, \rho)$.

In the case where $A(0)$ and $M(\Delta t)$ commute, precise measurements are characterized by the rms error-freeness condition, since in this case we have $\varepsilon_{G}(A(0), M(\Delta t))=\varepsilon(A, \rho)$. However, the probability reproducible condition does not characterize the precise measurements even in this case. To see this suppose that $A(0)$ and $M(\Delta t)$ are identically distributed and independent ${ }^{34}$ (p. 763). Then, we have

$$
\begin{aligned}
& \varepsilon_{G}(A(0), M(\Delta t))=\iint_{\mathbb{R}^{2}}(y-x)^{2} \mu^{A(0)}(d x) \mu^{M(\Delta t)}(d y) \\
& =\sigma(A(0))^{2}+\sigma(M(\Delta t))^{2}+(\langle A(0)\rangle-\langle M(\Delta t)\rangle)^{2} .
\end{aligned}
$$

Since $\sigma(A(0))=\sigma(M(\Delta t))$ and $\langle A(0)\rangle=\langle M(\Delta t)\rangle$, we have

$$
\varepsilon_{G}(A(0), M(\Delta t))=\sqrt{2} \sigma(A)
$$

Thus, $\mathbf{M}$ is not a precise measurement for the input state $\rho$ with $\sigma(A) \neq 0$. In the case where $A(0)$ and $M(\Delta t)$ do not commute, the rms error-freeness condition well characterizes precise measurements to a similar extent to the probability reproducibility condition. In particular, the class of measuring processes precisely measuring $A$ in all $\rho$ is characterized by the following equivalent conditions 33,34 : (i) $\varepsilon(A, \psi)=0$ for all $\psi \in \mathscr{H}$; (ii) probability reproducible for $A$ in all $\psi \in \mathscr{H}$; (iii) $\Pi=E^{A}$. The above result ensures our long-standing belief that a measurement with POVM $\Pi$ satisfying $\Pi=E^{A}$ is considered to be precise in any state in the sense that the measured observable $A(0)$ and the meter observable $M(\Delta t)$ to be directly observed are perfectly correlated in any input state, not only reproducing the probability distribution in any state.

We say that the measuring process $\mathbf{M}$ does not disturb an observable $B$ in a state $\rho$ iff observables $B(0)$ and $B(\Delta t)$ commute in the state $\rho \otimes \rho_{0}$ and the JPD $\mu^{B(0), B(\Delta t)}$ of $B(0)$ and $B(\Delta t)$ concentrates on the diagonal set. The non-disturbing measuring processes defined above can be characterized analogously.

From the above results, a non-zero lower bound for $\varepsilon(A)$ or $\eta(B)$ indicates impossibility of precise or non-disturbing measurement. In particular, if $\sigma(A), \sigma(B)<\infty$ and $\langle[A, B]\rangle \neq 0$, then any measuring process cannot precisely measure $A$ without disturbing $B$.

The above characterizations of precise and non-disturbing measurements suggests the following definitions of the locally uniform rms error $\bar{\varepsilon}(A, \rho)$ and the locally uniform rms disturbance $\bar{\eta}(B, \rho) 56$ :

$$
\begin{aligned}
& \bar{\varepsilon}(A, \rho)=\sup _{\phi \in \mathscr{C}(A, \rho)} \varepsilon(A, \phi), \\
& \bar{\eta}(B, \rho)=\sup _{\phi \in \mathscr{C}(B, \rho)} \eta(B, \phi) .
\end{aligned}
$$

Then, we have $\bar{\varepsilon}(A, \rho)=0$ if and only if the measurement precisely measures $A$ in $\rho$, and that $\bar{\eta}(B, \rho)=0$ if and only if the measurement does not disturb $B$ in $\rho$. For those quantities, the Heisenberg type EDR

$$
\bar{\varepsilon}(\hat{x}) \bar{\eta}\left(\hat{p}_{x}\right) \geq \frac{\hbar}{2}
$$

is still violated by a linear position measurement ${ }^{56}$, and the relation

$$
\bar{\varepsilon}(A) \bar{\eta}(B)+\bar{\varepsilon}(A) \sigma(B)+\sigma(A) \bar{\eta}(B) \geq \frac{1}{2}|\langle[A, B]\rangle|
$$

holds universally $\underline{56}$, where $\bar{\varepsilon}(A)=\bar{\varepsilon}(A, \rho)$ and $\bar{\eta}(B)=$ $\bar{\eta}(B, \rho)$.

Thus, the locally uniform rms error $\bar{\varepsilon}(A, \rho)$ completely characterizes precise measurements of $A$ in $\rho$ and the locally uniform rms disturbance $\bar{\eta}(B, \rho)$ completely characterizes measurements non-disturbing $B$ in $\rho$, while they satisfy the EDR of the same form as the rms error and disturbance. Further investigations on quantum generalizations of the classical notion of root-mean-square error and EDRs formulated with those quantities will be reported elsewhere.

\section{ACKNOWLEDGMENTS}

This work was supported in part by JSPS KAKENHI, No. 26247016 and No. 15K13456, and the John Templeton Foundation, ID \#35771.

\footnotetext{
* ozawa@is.nagoya-u.ac.jp

1 Heisenberg, W. The physical content of quantum kinematics and
}

mechanics. In Wheeler, J. A. \& Zurek, W. H. (eds.) Quantum Theory and Measurement, 62-84 (Princeton UP, Princeton, NJ, 
1983). [Originally published: Z. Phys., 43, 172-98 (1927)].

2 Kennard, E. H. Zur Quantenmechanik einfacher Bewegungstypen. Z. Phys. 44, 326-352 (1927).

3 von Neumann, J. Mathematical Foundations of Quantum Mechanics (Princeton UP, Princeton, NJ, 1955). [Originally puglished: Mathematische Grundlagen der Quantenmechanik (Springer, Berlin, 1932)].

4 Bohm, D. Quantum Theory (Prentice-Hall, New York, 1951).

5 Messiah, A. Mécanique Quantique, vol. I (Dunod, Paris, 1959). [Quantum Mechanics, Vol. I (North-Holland, Amsterdam, 1959)].

6 Schiff, L. I. Quantum Mechanics (MacGraw-Hill, New York, 1968).

7 Ballentine, L. E. The statistical interpretation of quantum mechanics. Rev. Mod. Phys. 42, 358-381 (1970).

8 Schrödinger, E. Die gegenwärtige Situation in der Quantenmechanik. Naturwissenshaften 23, 807-812, 823-828, 844-849 (1935). [English translation by J. D. Trimmer, Proc. Am. Philos. Soc. 124, 323-338 (1980)].

9 Braginsky, V. B. \& Khalili, F. Y. Quantum Measurement (Cambridge UP, Cambridge, 1992).

10 Giovannetti, V., Lloyd, S. \& Maccone, L. Quantum-enhanced measurements: Beating the standard quantum limit. Science 306, 1330-1336 (2004).

11 Ozawa, M. Universally valid reformulation of the Heisenberg uncertainty principle on noise and disturbance in measurement. Phys. Rev. A 67, 042105 (2003).

12 Ozawa, M. Uncertainty principle for quantum instruments and computing. Int. J. Quant. Inf. 1, 569-588 (2003).

13 Ozawa, M. Uncertainty relations for noise and disturbance in generalized quantum measurements. Ann. Phys. (N.Y.) 311, 350416 (2004).

14 Halmos, P. R. Introduction to Hilbert Space and the Theory of Spectral Multiplicity (Chelsea, New York, 1951).

15 Imoto, N., Ueda, M. \& Ogawa, T. Microscopic theory of the continuous measurement of photon number. Phys. Rev. A 41, 41274130 (1990).

16 Ozawa, M. Quantum measuring processes of continuous observables. J. Math. Phys. 25, 79-87 (1984).

17 Ozawa, M. Conditional probability and a posteriori states in quantum mechanics. Publ. Res. Inst. Math. Sci., Kyoto Univ. 21, 279 295 (1985)

18 Srinivas, M. D. Collapse postulate for observables with continuous spectra. Commun. Math. Phys. 71, 131-158 (1980).

19 Ozawa, M. Measuring processes and repeatability hypothesis. In Watanabe, S. \& Prohorov, Y. V. (eds.) Probability Theory and Mathematical Statistics, Lecture Notes in Math. 1299, 412-421 (Springer, Berlin, 1988).

20 Davies, E. B. \& Lewis, J. T. An operational approach to quantum probability. Commun. Math. Phys. 17, 239-260 (1970).

21 Helstrom, C. W. Quantum Detection and Estimation Theory (Academic, New York, 1976).

22 Ozawa, M. Conditional expectation and repeated measurements of continuous quantum observables. In Itô, K. \& Prohorov, J. V. (eds.) Probability Theory and Mathematical Statistics, Lecture Notes in Math. 1021, 518-525 (Springer, Berlin, 1983).

23 Reed, M. \& Simon, B. Methods of Modern Mathematical Physics, I: Functional Analysis (Revised and Enlarged Edition) (Academic, New York, 1980).

24 Gauss, C. F. Theory of the Combination of Observations Least Subject to Errors: Part One, Part Two, Supplement (SIAM, Philadelphia, USA, 1995). [Originally published: Theoria Combinationis Observationum Erroribus Miinimis Obnoxiae, Pars Prior, Pars Posterior, Supplementum (Societati Regiae Scientiarum Exhibita, Feb. 15, 1821)].
25 Braginsky, V. B., Vorontsov, Y. I. \& Thorne, K. S. Quantum nondemolition measurements. Science 209, 547-557 (1980).

26 Caves, C. M., Thorne, K. S., Drever, R. W. P., Sandberg, V. D. \& Zimmermann, M. On the measurement of a weak classical force coupled to a quantum mechanical oscillator, I, Issues of principle. Rev. Mod. Phys. 52, 341-392 (1980).

27 Caves, C. M. Defense of the standard quantum limit for free-mass position. Phys. Rev. Lett. 54, 2465-2468 (1985).

28 Yuen, H. P. Contractive states and the standard quantum limit for monitoring free-mass positions. Phys. Rev. Lett. 51, 719-722 (1983).

29 Ozawa, M. Measurement breaking the standard quantum limit for free-mass position. Phys. Rev. Lett. 60, 385-388 (1988).

30 Ozawa, M. Realization of measurement and the standard quantum limit. In Tombesi, P. \& Pike, E. R. (eds.) Squeezed and Nonclassical Light, 263-286 (Plenum, New York, 1989). ArXiv:1505.01083 [quant-ph]

31 Ozawa, M. Position measuring interactions and the Heisenberg uncertainty principle. Phys. Lett. A 299, 1-7 (2002).

32 Gudder, S. Joint distributions of observables. J. Math. Mech. 18, 325-335 (1968).

33 Ozawa, M. Perfect correlations between noncommuting observables. Phys. Lett. A 335, 11-19 (2005).

34 Ozawa, M. Quantum perfect correlations. Ann. Phys. (N.Y.) 321, 744-769 (2006).

35 Ozawa, M. Physical content of Heisenberg's uncertainty relation: limitation and reformulation. Phys. Lett. A 318, 21-29 (2003).

36 Arthurs, E. \& Goodman, M. S. Quantum correlations: A generalized Heisenberg uncertainty relation. Phys. Rev. Lett. 60, 24472449 (1988).

37 Raymer, M. G. Uncertainty principle for joint measurement of noncommuting variables. Am. J. Phys. 62, 986-993 (1994).

38 Ozawa, M. Quantum limits of measurements and uncertainty principle. In Bendjaballah, C., Hirota, O. \& Reynaud, S. (eds.) Quantum Aspects of Optical Communications, 3-17 (Springer, Berlin, 1991). ArXiv:1505.05083 [quant-ph].

39 Ishikawa, S. Uncertainty relations in simultaneous measurements for arbitrary observables. Rep. Math. Phys. 29, 257-273 (1991).

40 Ozawa, M. Uncertainty relations for joint measurements of noncommuting observables. Phys. Lett. A 320, 367-374 (2004).

41 Ozawa, M. Universal uncertainty principle in measurement operator formalism. J. Opt. B: Quantum Semiclass. Opt. 7, S672S681 (2005).

42 Ozawa, M. Conservation laws, uncertainty relations, and quantum limits of measurements. Phys. Rev. Lett. 88, 050402 (2002).

43 Ozawa, M. Universal uncertainty principle and quantum state control under conservation laws. AIP Conf. Proc. 734, 95-98 (2004).

44 Busch, P. \& Loveridge, L. Position measurements obeying momentum conservation. Phys. Rev. Lett. 106, 110406 (2011).

45 Wigner, E. P. Die Messung quntenmechanischer Operatoren. Z. Phys. 133, 101-108 (1952).

46 Araki, H. \& Yanase, M. M. Measurement of quantum mechanical operators. Phys. Rev. 120, 622-626 (1960).

47 Yanase, M. M. Optimal measuring apparatus. Phys. Rev. 123, 666-668 (1961).

48 Ozawa, M. Does a conservation law limit position measurements? Phys. Rev. Lett. 67, 1956-1959 (1991).

49 Busch, P., Heinonen, T. \& Lahti, P. Noise and disturbance in quantum measurement. Phys. Lett. A 320, 261-270 (2004).

50 Dressel, J. \& Nori, F. Certainty in heisenberg's uncertainty principle: Revisiting definitions for estimation errors and disturbance. Phys. Rev. A 89, 022106 (2014).

51 Korzekwa, K., Jennings, D. \& Rudolph, T. Operational 
constraints on state-dependent formulations of quantum errordisturbance trade-off relations. Phys. Rev. A 89, 052108 (2014).

52 Busch, P., Lahti, P. \& Werner, R. F. Colloquium: Quantum rootmean-square error and measurement uncertainty relations. Rev. Mod. Phys. 86, 1261-1281 (2014).

53 Lund, A. P. \& Wiseman, H. M. Measuring measurementdisturbance relationships with weak values. New J. Phys. 12, 093011 (2010).

54 Garretson, J. L., Wiseman, H. M., Pope, D. T. \& Pegg, D. T. The uncertainty relation in 'which-way' experiments: how to observe directly the momentum transfer using weak values. J. Opt. B: Quantum Semiclass. Opt. 6, S506-S517 (2004).

55 Mir, R. et al. A double-slit 'which-way' experiment on the complementarity-uncertainty debate. New J. Phys. 9, 287 (2007).

56 Ozawa, M. Noise and disturbance in quantum measurements and operations. Proc. SPIE 6244, 62440Q (2006). 